RESEARCH ARTICLE

\title{
Evaluation of Oxidative Stress Biomarkers in Patients after Bariatric Surgery of the Fobi Capella Type
}

\author{
Renata Pereira Simplício Lopes ${ }^{1}$ and Alexandre Coelho Serquiz ${ }^{2 *}$ \\ ${ }^{1}$ Undergraduate Student of Nutrition Course, University Center of Rio Grande do Norte, Brazil \\ ${ }^{2}$ Teacher of Nutrition Course, University Center of Rio Grande do Norte, Brazil
}

*Corresponding author: Alexandre Coelho Serquiz, Teacher of Nutrition Course, University Center of Rio Grande do Norte, Brazil

\begin{abstract}
Obesity is considered an increasing problem in the worldwide population, reaching epidemic proportions of about 400 million obese people in the world. The accumulation of adipose tissue leads to chronic oxidative stress, since its excess is associated with the development of inflammation, increasing the probability of developing comorbidities due to weight gain. This research aimed to evaluate the changes in inflammatory and antioxidants biomarkers after bariatric surgery in patients with morbid obesity. The study was executed with a population of 63 people with morbid obesity, composed of $77 \%$ of women and $23 \%$ of men, analyzing their food consumption, antioxidant level, oxidative stress, anthropometric and biochemical data. It was observed that after 6 months of surgical procedure, in addition to weight loss, there was a significant improvement in antioxidant biomarkers and oxidative stress.
\end{abstract}

\section{Keywords}

Obesity, Bariatric surgery, Oxidative stress, Antioxidants

\section{Introduction}

Obesity is characterized by the excessive accumulation of fat in the body, constituting a complex and multifactorial disease with severe social, psychological and clinical consequences, affecting all ages and social groups. Its prevalence has grown sharply in the last decades, being one of the most serious problems of public health. Overweight may be linked to an individual's genetic patrimony, poor eating habits, lifestyle, emotional factors or endocrine disorders, being a risk factor for a number of diseases [1].
The storage of adipose tissue is associated with the development of oxidative stress and inflammation, which can trigger comorbidities associated with this overweight, leading to risks in the development of chronic noncommunicable diseases (NCDs) such as type 2 diabetes and cardiovascular diseases which, if untreated, can lead to premature death or reduced quality of life. Visceral fat is an endocrine organ of hormonal storage and producer of inflammatory adipokines, causing the obese person to present chronic inflammation. Increased levels of IL-6 (interleukin-6) and TNF-alpha (tumor necrosis factor), multifunctional cytokines, are associated with morbidity and mortality [2].

Yet, the enzymes that act as markers of oxidative stress, CAT, SOD and NADPH, act by means of prevention mechanisms, preventing and/or controlling the formation of free radicals and non-radical species involved in the initiation of chain reactions that culminate in the propagation and amplification of the process and, consequently, the occurrence of oxidative damages [3].

Obesity can be treated through conservative, noninvasive therapies, aimed at modifying eating habits, with the follow-up of a nutritionist, followed by the stimulation of the practice of physical activity, in order to change the lifestyle. Also, in conjunction with these practices, the use of medications, which will be considered if there is failure associated with nonpharmacological treatment, if the patients have grade 2 and 3 obesity, or if they have any other obesity-related pathology [4].

Citation: Lopes RPS, Serquiz AC (2019) Evaluation of Oxidative Stress Biomarkers in Patients after Bariatric Surgery of the Fobi Capella Type. J Nutri Med Diet Care 5:032. doi.org/10.23937/25723278.1510032

Accepted: January 16, 2019: Published: January 18, 2019

Copyright: (c) 2019 Lopes RPS, et al. This is an open-access article distributed under the terms of the Creative Commons Attribution License, which permits unrestricted use, distribution, and reproduction in any medium, provided the original author and source are credited. 
Another therapy that has become increasingly frequent is non-conservative and invasive bariatric surgery (BS), and it has been shown to be efficient in reducing comorbidities associated with obesity, in weight loss, besides contributing to the improvement in quality of life. Surgical techniques differentiate according to the mechanism of action, being able to be restrictive, disabsorptive or mixed [5].

The technique of bariatric surgery discussed in the present study is the mixed one. It promotes a gastric reduction associated with malabsorption, especially the Roux-en-Y Gastric Bypass operation, or Fobi-Capella, which reduces stomach volume to $30 \mathrm{ml}$ [5].

Bariatric surgery is considered one of the most effective ways to treat severe obesity because, in addition to leading individuals to lose weight and possibly treat obesity-related comorbidities, it also enables a change in lifestyle and promotes new postsurgical habits [5].

Studies indicate that the reduction of obesity markers, which are the proinflammatory IL-6 (interleukin-6) and TNF-alpha (tumor necrosis factor), and those of oxidative stress, NADPH (nicotinamide adenine dinucleotide phosphate), CAT (catalase) and SOD (superoxide dismutase), bariatric surgery exerts a protective effect on the damage caused by oxidative stress [6].

Thus, the present study had as objective to evaluate the alterations of inflammatory and antioxidant biomarkers after bariatric surgery in patients with morbid obesity.

\section{Methodology}

\section{Type and place of study}

After approval by the research ethics committee, a clinical cohort study was applied, with sample selection by convenience. All patients underwent Fobi-Capella surgery and were invited to participate in the study; it was explained to them how the study would be performed and, after acceptance, the patient signed the TCLE (Informed Consent Form) (Appendix A). The study was conducted at a private bariatric surgery clinic in the city of Natal-RN, Brazil.

\section{Sample}

The sample was freely demanded according to the flow of surgeries in the clinic, in which the patients were in the pre-surgical phase and in the sixth postoperative month. The patients were submitted to Fobi-Capella technique, considered a mixed technique of surgery (restrictive and disabsorptive). The exclusion criteria considered were: smokers, alcoholics, use of antioxidants and patients with severe renal and hepatic diseases.

\section{Anamnesis}

In addition to chart analysis, patients were asked to fill in questionnaires containing cadastral data (name, age, gender, family per capita income, educational level, occupation) and clinical data (comorbidities, use of medications and/or supplements, consumption of antioxidant foods and the practice of physical activity) (Appendix B).

\section{Food consumption data}

Dietary intake was assessed by retrospective use, a 24-hour food recall (Appendix C), which was performed in the consultation before the surgical procedure and at the consultation 6 months after surgery. The quantities of food referred to by the patients, in a home measure, were converted to grams (g) or milliliters ( $\mathrm{ml}$ ) using a home measurement table (Appendix C). The consumption data were calculated by the TACO table (Tabela Brasileira de Composição de Alimentos - Brazilian Table of Food Composition). In order to evaluate the adequacy, the reference values of the Dietary Reference Intakes-DRI were used [7].

\section{Anthropometric data}

The anthropometric evaluation was performed before and after bariatric surgery, and the patients were weighed in a scale with the least possible clothing, barefoot, and in an erect position. The height was obtained by a stadiometer, the patient standing upright with the arms extended along the body, with his/her back to the wall, in the Frankfurt plane (Appendix B).

The BMI (Body Mass Index) was obtained according to the World Health Organization [8] for adults and Lipschitz [9] for the elderly.

CB measurement (arm circumference) was performed using an inelastic tape measure at the midpoint between the acromion and the olecranon. The TSF (triceps skinfold thickness) was measured by the adipometer at the back of the arm, also at the midpoint between the acromion and the olecranon. From the BS and TSF measurements, arm muscle circumference (AMC) was calculated, which is classified according to Frisancho [10].

For the measurement of AMP (adductor muscle thickness of the thumb), the patient maintained the hand relaxed, with the arm resting on the thigh, with the thumb away from the rest of the fingers, forming a $90^{\circ}$ angle with the index finger, so that the muscle could be pinched by the adipometer.

Three measurements of each variable were performed to obtain the average, as a final result.

\section{Evaluation of antioxidant and oxidative stress before and after bariatric surgery}

Approximately $5 \mathrm{ml}$ of blood was collected from the 
antecubital region of the forearm for analysis of the concentration of IL-6 (interleukin 6), TNF-alpha (tumor necrosis factor), SOD (superoxide dismutase) (16190SOD), CAT (catalase) (CAT100) and enzymatic cofactor NADP/ NADPH (nicotinamide adenine dinucleotide phosphate) (MAK038-1KT). The collections were performed by qualified individuals, and all biosafety procedures were considered. After the necessary preparations for each technique, the samples were frozen in an ultra-freezer at a negative temperature of $80^{\circ} \mathrm{C}$ degrees. Biochemical techniques were performed in the potiguar laboratory of Natal-RN, Brazil, and the dosage method used was Elisa (SIGMA-ALDRICHE, St. Louis-MO, USA).

\section{Biochemical evaluation}

Patients underwent routine biochemical exams and their results were taken from the medical records. Considering their importance, they are performed monthly, so that there is control in the levels of fasting glycemia, Total Cholesterol, HDL and Triglycerides.

\section{Statistical treatment}

All results were expressed as mean and standard deviation, structured and analyzed using descriptive analysis with the statistical package (PRISMA, 2017) version 7.0 for MacBook. Descriptive analysis of the general characteristics of the study population (sex, gender, weight, height, BMI, oxidative stress biomarkers and biochemical tests) and dietary parameters ( $24 \mathrm{~h}$ recall) were performed, and data were presented as mean \pm

Table 1: Profile of age and comorbidities of individuals undergoing bariatric surgery at a private clinic. $\mathrm{N}=63$ individuals.

\begin{tabular}{|l|l|}
\hline Patients $(\mathbf{n}=\mathbf{6 3})$ & Values \\
\hline Age & $37.4( \pm 10.61)$ \\
\hline Men & $23 \%$ \\
\hline Women & $77 \%$ \\
\hline Diabetics & $35 \%$ \\
\hline Hypertensive & $48.3 \%$ \\
\hline Dyslipidemia & $28.43 \%$ \\
\hline Apnea & $29 \%$ \\
\hline
\end{tabular}

standard deviation $(\mathrm{M} \pm \mathrm{SD})$. Student's t-test was performed. Statistical significance was defined as $p<0.05$.

\section{Results}

The study included 63 individuals who met the inclusion and exclusion criteria. The mean age was 37.4 $( \pm 10.61$ ) years, being composed predominantly by women (77\%). The most frequent comorbidities were systemic arterial hypertension (48.3\%) and diabetes mellitus (35\%), as shown in Table 1.

Regarding the anthropometric parameters, the individuals presented a reduction of $127 \mathrm{~kg}$ (kilogram) $( \pm 12.73)$ to $84.3 \mathrm{~kg}( \pm 12.93)$ in body weight $(p<0.05)$. As for fat mass and lean mass, a reduction of $51.4 \mathrm{~kg}$ (45-75) to $29.5 \mathrm{~kg}(21.5-49.5)$ was obtained with ( $p<$ $0.05)$, and a reduction of $56.4 \mathrm{~kg}(47-89.4)$ to $40.4 \mathrm{~kg}$ (31.8-82.2), respectively $(p<0.05)$. The initial BMI of $42.1 \mathrm{Kg} / \mathrm{m}^{2}(38.6-53.5)$ significantly reduced to $29.9 \mathrm{Kg} /$ $\mathrm{m}^{2}(26.4-39.8)(\mathrm{p}<0.001)$. A significant reduction in waist circumference variables was also observed in relation to the preoperative period (Table 2).

After 6 months of obesity surgery, subjects had a reduction in biochemical tests (glycemia, cholesterol and triglycerides) and had an increase in HDL parameters, as shown in Table 3.

Figure 1, shows the data of inflammatory markers. In the six-month period after obesity surgery, significant differences were obtained in inflammatory parameters $(p<0.0001)$, IL-6 with $268.6 \mathrm{pg} / \mathrm{mL}( \pm 18.5)$ and TNFalfa with $200.4 \mathrm{pg} / \mathrm{mL}( \pm 16.4)$. The results suggest that patients undergoing bariatric surgery significantly decreased inflammatory mediators after 6 months.

In Figure 2, oxidative stress was evaluated by the enzyme NADP/NADPH (nicotinamide adenine dinucleotide phosphate) that decreased after 6 months of bariatric surgery from $359.4 \mathrm{RLU} / \mathrm{mg}( \pm 53.5)$ before surgery to $249.3 \mathrm{RLU} / \mathrm{mg}( \pm 41.4)$ with significant difference $(p<0.0001)$.

Table 2: Anthropometric profile of individuals undergoing bariatric surgery at a private clinic. $\mathrm{N}=63$ individuals.

\begin{tabular}{|l|l|l|l|}
\hline Patients $(\mathbf{n}=\mathbf{6 3})$ & Before surgery & $\mathbf{6}$ months after surgery & p value \\
\hline Weight & $127( \pm 12.73)$ & $84.3( \pm 12.93)$ & $p<0.001$ \\
\hline BMI $\left(\mathrm{kg} / \mathrm{m}^{2}\right)$ & $42.1(38.6-53.5)$ & $29.9(26.4-39.8)$ & $p<0.001$ \\
\hline Fat mass $(\mathrm{Kg})$ & $51.4(45-75)$ & $29.5(21.5-49.5)$ & $p<0.001$ \\
\hline Lean mass $(\mathrm{Kg})$ & $56.4(47-89.4)$ & $40.4(31.8-82.2)$ & $p<0.05$ \\
\hline PC & $108.4( \pm 14.5)$ & $88.4( \pm 10.6)$ & $p<0.001$ \\
\hline
\end{tabular}

Table 3: Biochemical parameters of individuals undergoing bariatric surgery at a private clinic.

\begin{tabular}{|c|c|c|c|c|}
\hline Groups/Period & Before surgery & 6 months after surgery & $p$ value & Reference values \\
\hline Fasting glycemia (mg/dL) & $118.55( \pm 8.43)$ & $92.55( \pm 4.23)$ & $P<0.05$ & 70 to $99 \mathrm{mg} / \mathrm{dl}$ \\
\hline Total cholesterol (mg/dL) & $217.43( \pm 12.03)$ & $177.72( \pm 10.32)$ & $P<0.001$ & $\begin{array}{l}\text { Desirable: < than } 200 \mathrm{mg} / \mathrm{dl} \\
\text { Bordering: } 200 \text { to } 239 \mathrm{mg} / \mathrm{dl} \\
\text { High: Equal to or greater than } 240 \mathrm{mg} / \mathrm{dl}\end{array}$ \\
\hline $\mathrm{HDL}(\mathrm{mg} / \mathrm{dL})$ & $43.43( \pm 2.61)$ & $50.03( \pm 2.61)$ & $P<0.001$ & Equal to or greater than $40 \mathrm{mg} / \mathrm{dl}$ \\
\hline Triglycerides (mg/dL) & $228.55( \pm 16.29)$ & $123.68( \pm 9.34)$ & $P<0.001$ & $\begin{array}{l}\text { Man: } 3.5 \text { to } 7.2 \mathrm{mg} / \mathrm{dl} \\
\text { Woman: } 2.6 \text { to } 6.0 \mathrm{mg} / \mathrm{dl}\end{array}$ \\
\hline
\end{tabular}



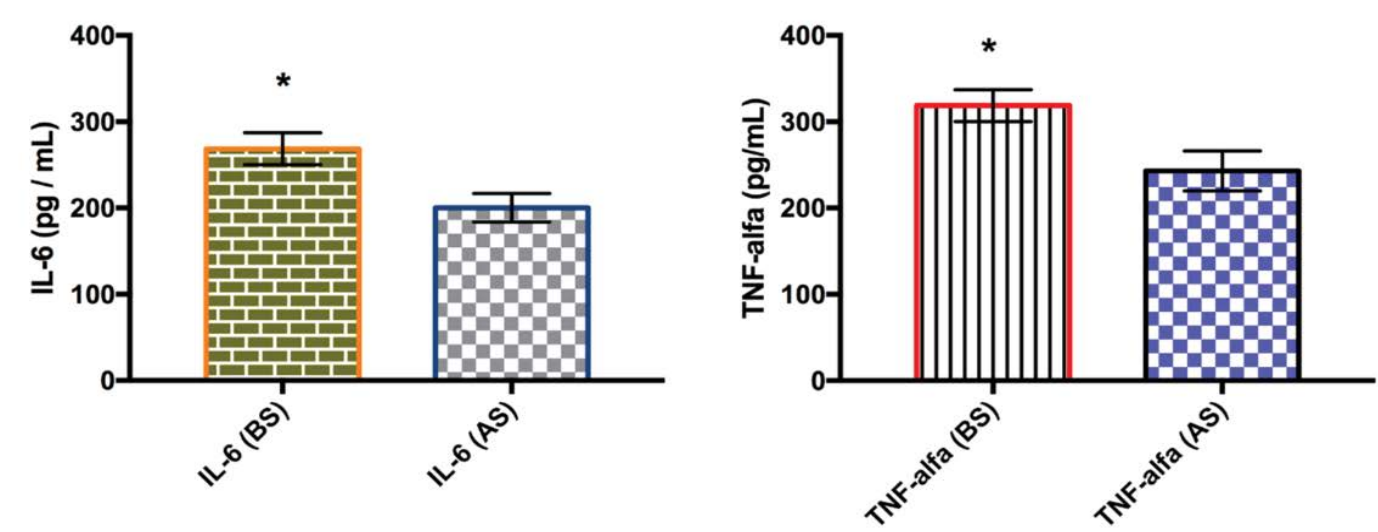

Figure 1: Evaluation of inflammatory markers of individuals undergoing bariatric surgery at a private clinic. BS: before surgery; AS: 6 months after surgery.
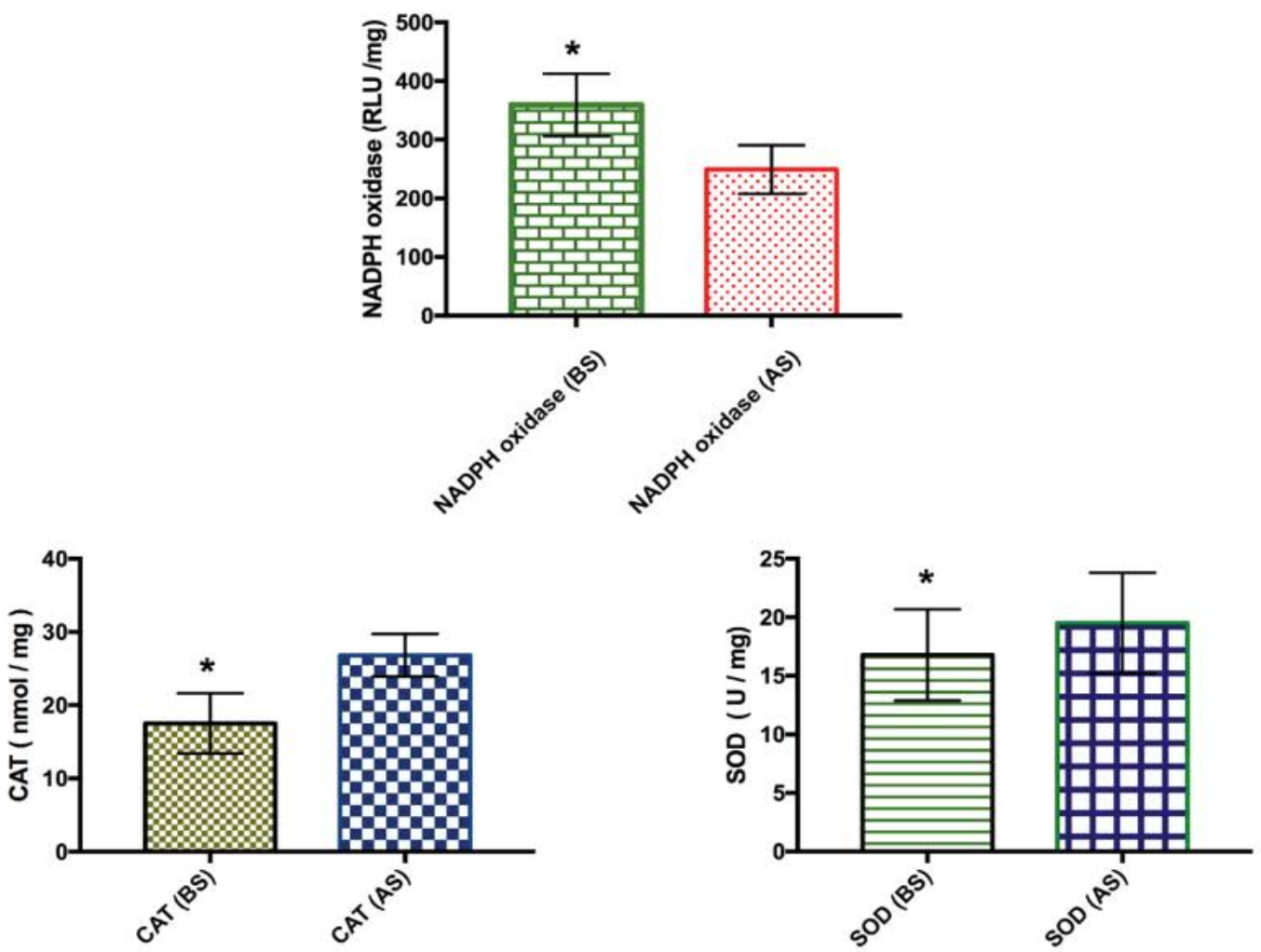

Figure 2: Evaluation of oxidative stress markers of individuals submitted to bariatric surgery at a private clinic. BS: before surgery; AS: 6 months after surgery.

Table 4: Correlation between IL-6 and TNF-alpha and the waist circumference.

\begin{tabular}{|l|l|l|}
\hline & \multicolumn{2}{|l|}{ IL-6 (after 6 months) } \\
\hline PC (after 6 months) & $r$ - Pearson & 0.824 \\
\cline { 2 - 3 } & $P$ value & $<0.0001$ \\
\hline & TNF-alpha (after 6 months) \\
\hline PC (after 6 months) & $r$ - Pearson & 0.678 \\
\hline & $P$ value & $<0.0001$ \\
\hline
\end{tabular}

As it can be observed in Figure 2, the CAT activities were significantly different between the groups ( $p<$ 0.05). Throughout the study, the activity of the CAT enzyme had an increase in the 6 months after the surgical procedure, showing its antioxidant potential, in relation to the basal moment. Regarding the SOD, the results were similar to those of CAT, for which 6 months after the surgical procedure there was a significant increase of the SOD enzyme $(p<0.05)$.

In Table 4, the correlations between IL-6 and TNFalpha with the waist circumference were evaluated. The results suggest a positive correlation between the parameters evaluated; the decrease in waist circumference (Table 2) decreases the inflammatory cytokines (Figure 1) in the individual after 6 months of bariatric surgery.

\section{Discussions}

Being overweight today is a global crisis. Today, $40 \%$ of the world's population is overweight, three times 
more than 40 years ago (World Health Organization) [11]. In Brazil, one in two adults, and one in three children, is in the same condition (Brazilian Institute of Geography and Statistics - IBGE, 2010). Obesity increases the risk of deadly diseases like diabetes, heart disease and at least 13 types of cancers, according to the National Cancer Institute (INCA) [12]. All these diseases are among the main causes of death in Brazil [13].

According to the Ministry of Health, in Brazil, at least $17 \%$ of the population is obese due to sedentary lifestyle and high consumption of industrialized products [14].

Due to the great increase in obesity indexes, there are currently several ways to fight it. Among the treatments, bariatric surgery stands out, which is an invasive procedure for individuals with class III obesity. This procedure has been developing technologically, and its advance is in accordance to the increase of the global epidemic of obesity. To date, only $1 \%$ of the patients undergoing this surgery have the effects of reversed weight loss [15].

According to the data described above, related to obesity and the performance of bariatric surgeries, the present study presents the benefits of this procedure, showing that individuals in the range of 37 years of age present significant changes in weight, with associated comorbidities, and that women are predominant in the number of bariatric surgeries. One possible explanation for this fact, perhaps, is because women are more concerned about beauty and health care [16].

The study points out that, of the 63 individuals who participated in the study, $48.3 \%$ were hypertensive and $35 \%$ were diabetic (Table 1), being these the most prevalent diseases due to obesity among patients. In a large cohort study carried out by Dra. Gunn Signe Jakobsen, doctor at morbid obesity center of the Sykehuset $i$ Vestfold in Tønsberg (Norway), it was seen that, in addition to weight loss, bariatric surgery also reduced the risk of hypertension and other obesityrelated comorbidities [17].

Table 2 of the present study shows that there was a very significant reduction of $p<0.001$ in body weight, in $\mathrm{BMI}$ and in the fat mass index of the patients, as well as a significant loss of $p<0.05$ of lean mass after 6 months of bariatric surgery; it was notorious that results related to weight loss associated with a change in body fat distribution allowed a considerable improvement in biochemical tests (Table 3), with a significant improvement in the markers of Glycemia, Total Cholesterol, HDL and Triglycerides, thus reducing the risk of diseases associated with obesity.

The possible explanation for this fact must be due to the significant decrease in the number of adipose tissue (Table 2), since the amount of fat is directly related to the increase in insulin resistance. The indicators of body composition are strongly associated with metabolic alterations, and it is important to verify their relation with the components of metabolic syndrome and insulin resistance [18], increase in blood pressure, since the excessive fat accumulation in the abdominal region and the deposition of adipose tissue in the viscera are strongly related to the cardiovascular risk factors [19].

Adipose tissue has the capacity to produce adipocytokines, substances that participate in the inflammatory response. These adipocytokines may present pro- or anti-inflammatory actions, such as TNF- $\alpha$, IL- 6 and adiponectin, respectively. Obese individuals have reduced levels of adiponectin [20].

Figure 1 shows the evaluation of inflammatory markers of patients undergoing bariatric surgery. Results were obtained with significant differences in the parameters of the proinflammatory cytokines IL- 6 (interleukin) and TNF- $\alpha$ (tumor necrosis factor) after 6 months of surgery, showing that the reduction of adipose tissue has a great impact on these biomarkers. In an article that addresses the inflammatory processes of obesity, it has been seen that adipose tissue is not just a stock organ. It also plays the role of producer of certain bioactive substances as IL-6, with inflammatory functions. As the amount of adipose cells increases, there is a change in the production of inflammatory adipokines, that is, the greater the amount of adipose tissue, the greater the secretion of these adipokines, such as TNF- $\alpha$ and IL-6. They are adipokines with immune function, and are produced by adipocytes in response to infectious or inflammatory stimuli. The authors concluded that reducing body mass through bariatric surgery is a safe method to decrease the inflammatory state of these biomarkers [21].

Thus, according to the benefits derived from the surgical intervention for the control and reversal of obesity, the evaluation of antioxidant biomarkers and oxidative stress in patients after Fobi Capella type bariatric surgery was exposed in Figure 2.

For a better understanding, it remains to be known that free radicals are reactive molecules containing a number of unpaired electrons, which are produced in the normal course of energy metabolism. According to this theory, toxic effects of these radicals are introduced into the cellular interior, triggering deleterious reactions called oxidative stress [22].

The installation of the process of oxidative stress arises from the existence of an imbalance between oxidant and antioxidants compounds, in favor of excessive generation of free radicals or to the detriment of their speed of removal. Such a process leads to the oxidation of biomolecules with consequent loss of their biological functions and/or homeostatic imbalance; the manifestation of which is potential oxidative damage against cells and tissues [3]. 
Obesity and associated comorbidities are one of the causes of this homeostatic imbalance, giving rise to oxidative damage [23].

In Figure 2, the results of oxidative stress biomarkers can be observed by the enzymes cofactor NADP/NADPH (nicotinamide adenine dinucleotide phosphate), CAT (catalase) and SOD (superoxide dismutase), which are natural enzyme defenses. It was observed that there was extreme significance $(p<0.001)$ in the biomarkers of NADPH, which is a pro-oxidant enzyme, and a reduction after 6 months of surgery was observed. With the CAT and SOD antioxidant enzymes, it was observed that there was a significant increase $(p<0.05)$ in biomarkers after 6 months of surgery, in relation to basal moment, leading to the belief in the benefits of bariatric surgery, and that fat reduction improved the picture of chronic diseases associated with obesity, in view of the balance between pro-oxidant and antioxidant enzymes.

In the present study, the results suggest that the surgical procedure promoted weight loss accompanied by the decrease of body fat, improving inflammation parameters.

The results also suggest that the decrease of the inflammatory cytokines has a relation with the decrease of visceral fat, as shown in Table 3 and in Figure 1. In the study by [24], it was demonstrated the body mass and the subsequent improvement of biochemical, metabolic and anthropometric parameters in patients undergoing bariatric surgery. This may contribute to the reduction of patient oxidation and, consequently, reduce the risks of development and multiple comorbidities associated with obesity.

Therefore, the results show that the surgical procedure promoted an improvement of the defense system (Figure 2), allowing the increase of the CAT and SOD enzymes, which constitute the first line of antioxidant defense and the largest defense system of the organism, as well as the decrease of NADPH, showing the reduction of oxidative stress [25].

\section{Final Considerations}

In analyzes performed in the data collections of the present study, a significant improvement was observed in all aspects after 6 months of bariatric surgery, such as weight reduction, improvement in biochemical tests, and a decrease in fasting glycemia, in total cholesterol, in $\mathrm{HDL}$ and in triglycerides, in inflammatory markers (IL-6 and TNF-alpha), as well as in oxidative stress markers (NADPH, CAT and SOD). This leads us to believe that bariatric surgery was of total relevance for an improvement in the quality of life of these patients. However, this is still a topic that needs to be discussed and studied, analyzing genetic and external factors, lifestyle, among others, since its prevention and final resolution are extremely important for the well-being of the population affected by obesity.

\section{References}

1. Barroso TA, Lucas Braga Marins, Renata Alves, Ana Caroline Souza Gonçalves, Sérgio Girão Barroso, et al. (2017) Association between central obesity and the incidence of diseases and cardiovascular risk factors. International Journal of Cardiovascular Sciences 30: 416424.

2. Patrícia Amante de Oliveira (2017) Inflammatory indicators, endothelial function and other markers of cardiac risk in elderly patients with overweight and obesity: Response to supplementation of olive oil, flaxseed oil and sunflower oil.

3. Barbosa KBF, Neuza Maria Brunoro Costa, Rita de Cássia Gonçalves Alfenas, Sérgio Oliveira De Paula, Valéria Paula Rodrigues Minim, et al. (2010) Oxidative stress: Concept, implications and modulatory factors. Rev Nutr 23: 629-643.

4. Costa AMJC, Duarte SFP (2017) Main medications used in the treatment of obesity and pathways of action: A systematic review. Id on Line Rev Psic 11.

5. Oliveira LSFO, Mauro Lúcio Mazini Filho, Gabriella Venturini, Juliana Brandão Pinto de Castro, Maria Elisa Caputo Ferreira (2018) Bariatric surgery repercussions on the quality of life of patients with obesity: An integrative review. Revista Brasileira de Obesidade, Nutrição e Emagrecimento 12: 47-58.

6. Neves, Cibele Ferreira (2010) Evaluation of biomarkers of oxidative stress and inflammatory status in patients before and after surgery of obesity.

7. IOM/Food and Nutrition Board (2002) Dietary reference intakes for energy, carbohydrate, fiber, fat, fatty acids, cholesterol, protein, and amino acids (Macronutrients). The National Academies Press.

8. World Health Organization (1995) Physical status: The use and interpretation of anthropometry. Geneva.

9. Lipschitz D (1997) To evaluate and treat nutritional problems in elderly patients. Clin Geriatr Med 13: 753-768.

10. Frisancho AR (1990) Anthropometric standards for the assessment of growth and nutritional status. University of Michigan Press, Ann Arbor, Michigan.

11. World Health Organization (2018) Obesity and overweight fact sheet.

12. Instituto Nacional de Câncer José Alencar Gomes da Silva (2017) Posicionamento do instituto nacional de câncer josé alencar gomes da silva acerca do sobrepeso e da obesidade.

13. Ana Paula Bortoletto Martins (2018) Need to treat obesity as a public health problem. Rev adm empres 58: 3 .

14. Letícia Carvalho Massaroli, Letícia Cristina Santos, Giovanna Gomes Carvalho, Stephanie Avesani João Figueiredo Carneiro, Laura Ferreira de Rezende (2018) Quality of life and high BMI as a risk factor for cardiovascular diseases: A systematic review. Revista da Universidade Vale do Rio Verde 16.

15. Anna Christina Charbel Costa, Maria Lucia Ivo, Wilson de Barros Cantero, Joao Ricardo Filgueiras Tognini (2009) Obesity in patients candidates for bariatric surgery. Acta Paul Enferm 22: 55-59.

16. Paola Turchiello da Silva, Luciana Dapieve Patias, Glauco da Costa Alvarez, Vanessa Ramos Kirsten, Elisângela Colpo, et al. (2015) Profile of patients seeking bariatric surgery. ABCD Arq Bras Cir Dig 28: 270-273.

17. Jakobsen GS, Milada Cvancarova Småstuen, Rune 
Sandbu, Njord Nordstrand, Dag Hofs $\varnothing$, et al. (2018) Association of bariatric surgery vs. medical obesity treatment with long-term medical complications and obesity-related comorbidities. JAMA 319: 291-301.

18. Gobato AO, Ana Carolina J Vasques, Mariana Porto Zambon, Antonio de Azevedo Barros Filho, Gabriel Hessel, et al. (2014) Metabolic syndrome and insulin resistance in obese adolescents. Rev Paul Pediatr 32: 55-62.

19. Pavanelli MF, Arantes VC, Ribas Silva RC (2012) Relationship between visceral adiposity and systemic arterial hypertension. Rev Enferm UFSM 2: 584-590.

20. Denise Nogueira Carvalho, Jomara Nogueira Carvalho, Jussara Gomes de Brito, Priscila da Silva Rocha (2016) Inflammatory aspects of adipose tissue in obesity. Journal of Amazon Health Science 2.

21. Sippel C, Rafaela Mundstock de Azevedo Bastianb, Janine Giovanellac, Christiane Faccind, Verônica Continie, et al. (2014) Inflammatory processes of obesity. Revista de
Atenção à Saúde 12: 48-56.

22. Antunes-Neto JMF, Silva LP, Macedo DV (2005) Oxidative stress biomarkers: New possibilities for monitoring in physical training. $\mathrm{R}$ bras $\mathrm{Ci}$ e Mov 13: 7-15.

23. Pedrosa IV, Maria Goretti Pessoa de Araújo Burgos, Niedja Cristina Souza, Caroline Neves de Morais (2009) Nutritional aspects in obese before and after bariatric surgery. Rev Col Bras Cir 36: 316-322.

24. Schmatz R, Mariana R Bitencourt, Luciana D Patias, Maristela Beck, Glaucoda C Alvarez, et al. (2017) Evaluation of the biochemical, inflammatory and oxidative profile of obese patients given clinical treatment and bariatric surgery. Clinica Chimica Acta 465: 72-79.

25. Soares ER, Elisa B Monteiro, Renata C da Silva, Aline Batista, Flavia Sobreira, et al. (2015) Bioactive compounds in food, oxidative stress and inflammation: A molecular view of nutrition. Revista Hospital Universitário Pedro Ernesto 14: 64-72. 